\title{
Role of PSA Kinetics in Hormone-refractory Prostate Cancer
}

\author{
Volkan Sabur ${ }^{1}$, Ibrahim Untan ${ }^{2}$ and Atila Tatlisen ${ }^{3}$ \\ ${ }^{1}$ Department of Urology, Erciyes Kartal Hospital, Kayseri, Turkey \\ ${ }^{2}$ Department of Urology, Ahi Evran University, Training and Research Hospital, Kirsehir, Turkey \\ ${ }^{3}$ Department of Urology, Erciyes University, Faculty of Medicine, Kayseri, Turkey
}

\begin{abstract}
Objective: To demonstrate the predictive effect of PSA derivatives and time markers that is prostate-specific antigen (PSA) doubling time (PSADT) and PSA velocity (PSAV) on survival in men with hormone-refractory prostate cancer (HRPCa).

Study Design: Descriptive, analytical study.

Place and Duration of Study: Department of Urology, Erciyes University, Faculty of Medicine, Kayseri, Turkey, between 2012 and 2020.

Methodology: One hundred patients, who were treated with prostate cancer, were subjected. The PSA values were noted, nadir PSA values were detected, times to nadir PSA, HRPCa, and follow-up times were recorded. PSADT and PSAV were calculated. The relationships between the groups were analyzed. Kaplan-Meier curves were used to estimate overall survival between the groups.

Results: The patients were grouped according to the mean PSADT, median PSAV, median nadir PSA, and mean time to HRPCA. The survival of those with high PSADT, low PSAV and low nadir PSA were found to be significantly longer ( $p=0.006, p<0.001$, $p<0.001)$. High PSAV was also associated with significantly increased PSA, nadir PSA and death $(p<0.001, p=0.47$, and $p<0.001, p=0.52$, respectively). The survival of those with a longer time to HPRCa was found to be significantly longer $(p<0.001)$. There was no statistically significant difference in terms of survival between patients who received chemotherapy after HRPCa and those who did not $(p=0.477)$. PSAV ( $p=0.007 \mathrm{HR}: 1.004$ 95\% Cl: $1.001-1.007)$, bone metastasis at diagnosis $(p=0.001 \mathrm{HR}: 0.35795 \% \mathrm{Cl}: 0.197-0.645)$ and time to HRPCa development ( $=0.001 \mathrm{HR}: 0.93695 \% \mathrm{Cl}: 0.900-0.974)$ were significantly effective to the survival.

Conclusion: PSADT, PSAV, and nadir PSA serve as independent prognostic markers for survival in patients with HRPCa. These three PSA derived calculation products, with the help of other parameters, could work as prognostic factors, and help clinicians predict survival in men with HRPCa.
\end{abstract}

Key Words: PSA kinetics, Hormone-refractory prostate cancer, Survival, Prognostic factors.

How to cite this article: Sabur V, Untan I, Tatlisen A. Role of PSA Kinetics in Hormone-refractory Prostate Cancer. J Coll Physicians Surg Pak 2021; 31(06):673-678.

\section{INTRODUCTION}

Nowadays, prostate cancer, is ranked first in men in developed western countries, and second in Turkey. ${ }^{1}$ Prostate cancer has a strong relationship with aging. ${ }^{2}$ Although the survival rate is high for early-stage prostate cancer, however, there is no adequate salvage therapy for the relapsing disease. ${ }^{3}$ After hormone treatments at advanced stages, prostate cancer almost always becomes hormone-resistant. ${ }^{4}$

Correspondence to: Dr. Ibrahim Untan, Department of Urology, Ahi Evran University, Training and Research Hospital, Kirşehir, Turkey

E-mail: ibrahimuntan@erciyes.edu.tr

Received: March 01, 2021; Revised: April 28, 2021;

Accepted: April 28, 2021

DOI: https://doi.org/10.29271/jcpsp.2021.06.673
Despite advances in the treatment of HRPCa, its mortality is still very high. ${ }^{5}$ The main purpose of the treatment is to increase the survival time and to provide symptomatic improvement with palliative treatment. Progression and death are inevitable with the treatments currently being applied for patients with HRPCa. ${ }^{6}$

PSA is an important marker that is used in the diagnosis, treatment planning, and follow-up of prostate cancer. However, due to its low specificity, it is insufficient in follow-up and predicting relapse, as it is in diagnosis, and it does not contribute significantly to survival. Moreover, since it is organ-specific rather than disease-specific, it is not considered an ideal tumor marker. The positive predictive value of PSA is $42 \%$, and when used with the digital rectal examination, this value increases to $60 \%$ to diagnose prostate cancer. ${ }^{7}$ Because of this diagnostic deficiency of PSA, it has been suggested to use different criteria such as PSA density, PSA velocity (PSAV), PSA doubling time (PSADT), free/total PSA, and complex PSA. 
In this study, locally advanced or advanced-stage patients, who were diagnosed with prostate cancer and given androgen ablation therapy, were examined. It was aimed to evaluate PSADT, PSAV, and certain other indicators on survival after the development of hormone-resistant cancer in men. In this context, if these indicators mentioned prove themselves as a prognostic marker, it is thought that patients with locally advanced or advanced-stage prostate cancer may provide the advantage of making more accurate decisions about the need and timing of adjuvant treatment by making more careful follow-up after the developmentof hormone-resistantcancer.

The objective of this study was to demonstrate the predictive effect of PSA derivatives and time markers that is prostate-specific antigen (PSA) doubling time (PSADT) and PSA velocity (PSAV) on survival in men with hormone-refractory prostate cancer(HRPCa).

\section{METHODOLOGY}

The study was conducted in Department of Urology, Erciyes University, Faculty of Medicine, between 2012 and 2020. One hundred patients who were admitted with a diagnosis of locally advanced or advanced-stage prostate cancer and who received only hormonal therapy as the initial treatment and later developed resistance to hormonal therapy, and who had sufficient medical records, were included in the study.

Androgen deprivation therapy was applied to locally advanced or advanced-stage patients. It was determined whether the hormonal treatment modalities applied to these patients were surgical (bilateral orchiectomy) or medical (Luteinising hormone-releasing hormone analogs or antiandrogens) castration. After reaching the lowest PSA value (nadir PSA) during hormonal therapy, when PSA elevation is detected in their followup if they are not already taking, antiandrogens were added to the patients' treatment. On the other hand, antiandrogens were withdrawn in patients receiving antiandrogen therapy. Nevertheless, measuring serum testosterone level below $50 \mathrm{ng} / \mathrm{ml}$, detection of at least two rising PSA values measured every one month and the measured PSA values exceeding $50 \%$ of nadir PSA, recognition of new metastases that clinically indicate cancer progression were considered as HRPCa diagnosed. Progression of lesions or two or more new lesions occurring in the bone scan, soft tissue lesions, or having lymph nodes larger than two $\mathrm{cm}$ according to response evaluation criteria in solid tumours (RECIST) were accepted as progression. ${ }^{8}$

In all patients, after the development of HRPCa, the first three PSA values measured 1 - 3 months apart were recorded in the period when no additional treatment was given. Using these PSA values, PSADT and PSAV were calculated.

Patients' age at the time of diagnosis, clinical stages, Gleason scores, treatment modalities, PSA values at the time of diagnosis, bone metastasis and lymph node involvement at the time of diagnosis, nadir PSA values, time to nadir PSA, and time to HRPCa development, chemotherapy status, follow-up dura- tions, death status, and PSADT and PSAV values were recorded. The correlation between PSADT and PSAV and other predictive values were compared and the relationship between them and survival was investigated.

IBM SPSS version 22.0 (IBM Corp. Released 2013. IBM SPSS Statistics for Windows, Version 22.0 Armonk, NY: IBM Corp.) was used for statistical analysis of the datasets. Quantitative data were expressed as mean $( \pm S d)$ and median $(I Q R)$. The compliance of the data to normal distribution was tested with the Shapiro-Wilktest. Comparisons between groups wereevaluated using the Mann-Whitney U-test. Spearman test was used to determine the relationships between variables. KaplanMeier analysis was used for survival analysis and Cox regression analysis was used to determine risk factors. A significance level of $p<0.05$ was accepted.

\section{RESULTS}

The mean age of 100 patients included in the study was 69.36 ( \pm 7.46). PSA before treatment, Gleason score, nadir PSA, time to nadir PSA, time to HRPCa, follow-up time after diagnosis, PSADT and PSAV calculated after PSA relapse were the parameters analysed. All variables except age were non-normally distributed. It was observed that 48 of these patients had bone metastasis and 33 had lymph node involvement at the time of diagnosis. Twenty-three patients received chemotherapy after the diagnosis of HRPCa. 54 of all patients died during the followup period. Patients' descriptives can be viewed in Tablel.

\section{Table I: Patients' descriptions.}

\begin{tabular}{|l|c|c|c|}
\hline $\mathrm{n}=100$ & Unit & Mean $( \pm \mathrm{Sd})$ & Median $\left(25^{\text {th }}-75^{\text {th }}\right)$ \\
\hline Age & years & $69.36( \pm 7.46)$ & $70(65.25-74.00)$ \\
\hline Diagnose PSA & $\mathrm{ng} / \mathrm{ml}$ & $104.56( \pm 139.99)$ & $67(24.77-118.52)$ \\
\hline Gleason score & Score total & $8.04( \pm 1.27)$ & $8(7-9)$ \\
\hline Nadir PSA & $\mathrm{ng} / \mathrm{ml}$ & $2.38( \pm 3.12)$ & $1.46(0.51-2.71)$ \\
\hline Time to nadir & months & $9.16( \pm 6.72)$ & $7(5.00-11.75)$ \\
\hline Time to HRPca & months & $17.13( \pm 11.90)$ & $13(10-20)$ \\
\hline Follow up time & months & $39.38( \pm 22.47)$ & $36(23.25-52.50)$ \\
\hline PSADT & months & $4.23( \pm 2.96)$ & $3.59(2.33-4.89)$ \\
\hline PSAV & ng/ml/years & $78.59( \pm 91.26)$ & $35.82(14.12-101.26)$ \\
\hline
\end{tabular}

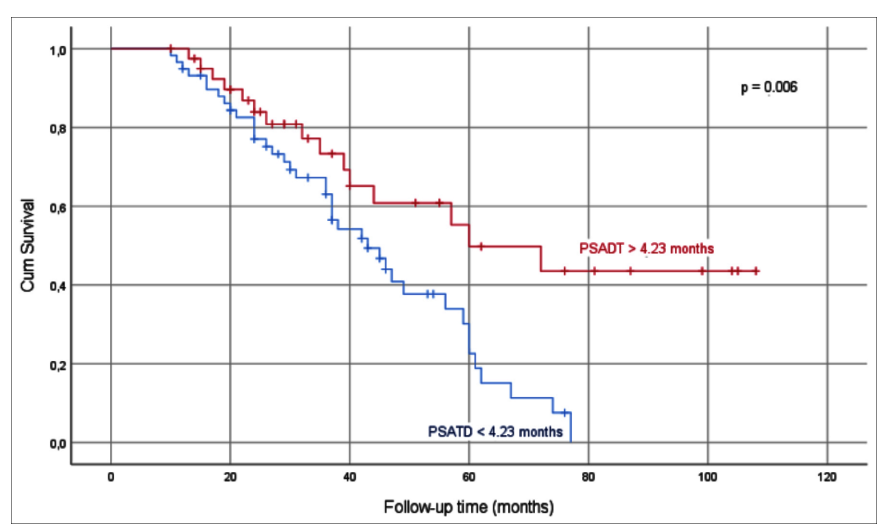

Figure 1: Kaplan-Meier survival analysis in groups divided according to PSADT mean value. 
Table II: Associations between groups divided according to PSADT mean and PSAV median values and patients' descriptions.

\begin{tabular}{|c|c|c|c|c|c|c|}
\hline & PSADT $<4.23$ & PSADT > 4.23 & & PSAV < 35.82 & PSAV > 35.82 & \\
\hline & median $\left(25^{\text {th }}-75^{\text {th }}\right)$ & median $\left(25^{\text {th }}-75^{\text {th }}\right)$ & $\mathrm{p}$ & median $\left(25^{\text {th }}-75^{\text {th }}\right)$ & median $\left(25^{\text {th }}-75^{\text {th }}\right)$ & $\mathrm{p}$ \\
\hline Age & $67(63-72)$ & $72(69-75.50)$ & 0.001 & $71(67-75)$ & $68(64-72.25)$ & 0.099 \\
\hline Diagnose PSA & $68(25.45-126)$ & $45(23.78-104.50)$ & 0.309 & $36.65(20.77-100)$ & $100(44.50-140)$ & 0.001 \\
\hline Gleason score & $8(7-9)$ & $9(8-9)$ & 0.220 & $8(7-9)$ & $8(7-9)$ & 0.614 \\
\hline Nadir PSA & $1.28(0.52-2.50)$ & $1.60(0.45-3.37)$ & 0.629 & $0.74(0.18-1.60)$ & $2.15(1.22-4.72)$ & $<0.001$ \\
\hline Time to nadir & $7(4-12)$ & $7(5-11.50)$ & 0.574 & $8(5-12)$ & $7(4-11.25)$ & 0.226 \\
\hline Time to HRPca & $13(8-19)$ & $14(10.25-24.25)$ & 0.284 & $13(11.50-27.50)$ & $13.50(8-18.50)$ & 0.139 \\
\hline Follow-up time & $36(24-46)$ & $33(22.50-58.50)$ & 0.629 & $37(23.50-54.25)$ & $31(22.50-50)$ & 0.436 \\
\hline PSADT & $2.63(1.88-3.35)$ & $5.63(4.54-7.26)$ & $<0.001$ & $3.91(2.91-5.38)$ & $3.18(1.85-4.53)$ & 0.007 \\
\hline PSAV & $59.38(26.69-188.55)$ & $15.11(8.45-52.14)$ & $<0.001$ & $14.42(9.49-26.91)$ & $99.53(56.75-207.72$ & $<0.001$ \\
\hline
\end{tabular}

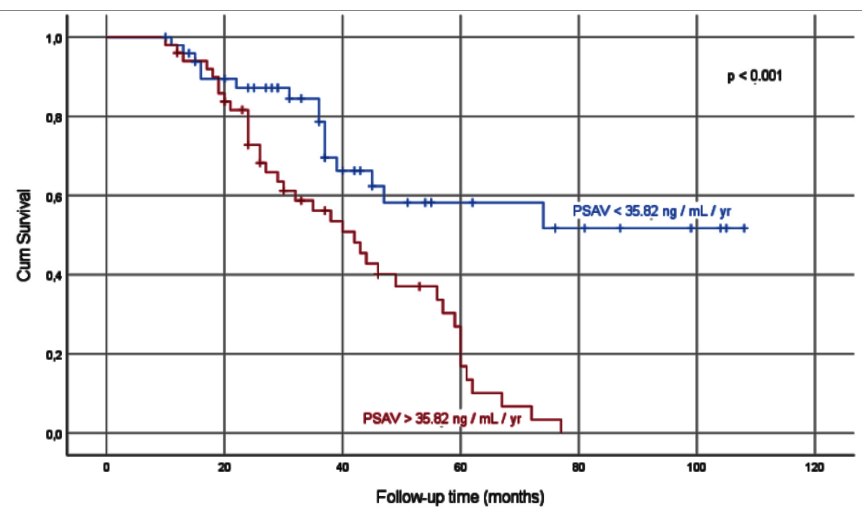

Figure 2: Kaplan-Meier survival analysis in groups divided according to PSAV median value.

Table III: Cox regression analysis of the variables.

\begin{tabular}{|l|c|c|c|c|c|c|}
\hline & \multicolumn{3}{|c|}{ univariate } & \multicolumn{3}{c|}{ multivariate } \\
\hline Variables & $\mathrm{HR}$ & $\% 95 \mathrm{Cl}$ & $\mathrm{p}$ & $\mathrm{HR}$ & $\% 95 \mathrm{Cl}$ & $\mathrm{p}$ \\
\hline Diagnose PSA & 1.004 & $1.002-1.006$ & $<0.001$ & - & - & - \\
\hline Gleason score & 1.250 & $1.009-1.549$ & 0.041 & - & - & - \\
\hline Nadir PSA & 1.082 & $1.011-1.159$ & 0.024 & - & - & - \\
\hline Bone metastasis & 3.839 & $2.144-6.874$ & $<0.001$ & 0.357 & $\begin{array}{c}0.197- \\
0.645\end{array}$ & 0.001 \\
\hline Time to HRPca & 0.935 & $0.903-0.968$ & $<0.001$ & 0.936 & $\begin{array}{c}0.900- \\
0.974\end{array}$ & 0.001 \\
\hline PSADT & 0.863 & $0.762-0.978$ & 0.021 & - & - & - \\
\hline PSAV & 1.006 & $1.003-1.008$ & $<0.001$ & 1.004 & $\begin{array}{c}1.001- \\
1.007\end{array}$ & 0.007 \\
\hline
\end{tabular}

The patients were divided into two groups as those with a PSADT above and below 4.23 months which is the mean value of PSADT. There were 59 patients in PSADT $<4.23$ group and 41 patients in PSADT $>4.23$ group (Table II). The life span of the PSADT $>4.23$ group was significantly longer $(p=0.006$, Figure 1$)$.

The patients were divided into two groups as those with a PSAV above and below $35.82 \mathrm{ng} / \mathrm{ml} / \mathrm{year}$ which is the median value of PSAV. Both groups had 50 patients (Table II). The follow-up time of the PSAV $<35.82 \mathrm{ng} / \mathrm{ml} /$ year group was significantly longer $(p<0.001$, Figure 2$)$.

In the Spearman correlation test, a significant correlation was found between diagnostic PSA and PSAV, lymph node involvement on diagnosis, and bone metastasis on diagnosis $(p<0.001, p=0.40, p<0.001, p=0.41$, and $p<0.001$, $\rho=0.54$, respectively). A significant correlation was discovered between PSAV and nadir PSA, and death $(p<0.001$, $\rho=0.47$, and $p<0.001, \rho=0.52$, respectively). Time to nadir PSA showed a correlation with time to HRPCa and follow-up time $(p<0.001, p=0.77, p<0.001$, and $p=0.42$, respectively). Metastasis on prognosis found to be associated with progression during follow-up $(p<0.001, \rho=0.48)$. A correlation between PSADT and PSAV, in each statistical test is an expected result since these are computationally obtained values derived from PSA.

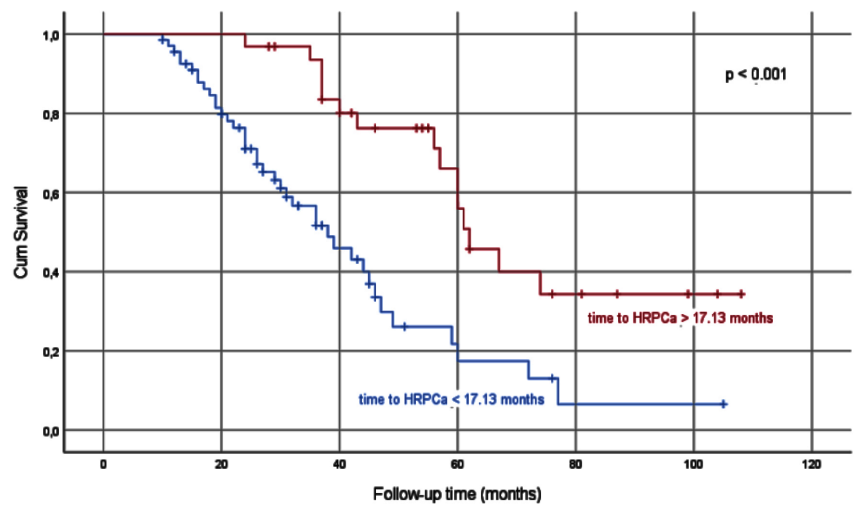

Figure 3: Kaplan-Meier survival analysis in groups divided according to time to HRPCa mean value.

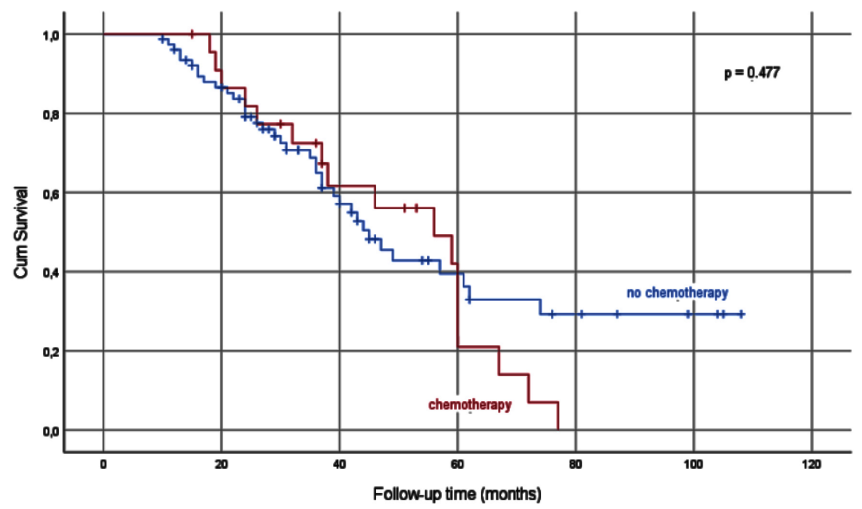

Figure 4: Kaplan-Meier survival analysis in groups divided according to recieving chemotherapy or not.

The patients were divided into two groups as those with a nadir PSA above and below $1.46 \mathrm{ng} / \mathrm{ml}$ which is the median value of nadir PSA. There were 49 patients in nadir PSA $<1.46$ group and 51 patients in nadir PSA $>1.46$ group. The survival 
of the nadir PSA $<1.46$ group was statistically longer than the survival of the nadir PSA $>1.46$ group (Kaplan- Meier value $p<0.001$, Mann-Whitney $U$ value $p=0.003$ ).

The patients were divided into two groups as those with a time to HRPCa above and below 17.13 months, which is the mean value of time to HRPCa. There were 68 patients in time to HRPCa $<17.13$ months group and 32 patients in time to HRPCa $<17.13$ months group. The survival of the time to HRPCa > 17.13 months group was statistically longer than the survival of the time to HRPCa $<17.13$ months group ( $p$ $<0.001$, Figure 3). Patients who received and did not receive chemotherapy were analyzed in terms of survival. There were 32 patients in the chemotherapy group and 77 in the no chemoterapy group. It was observed that there was no statistically significant difference in terms of survival between patients who received chemotherapy after HRPCa and those who did not $(p=0.477$, Figure 4$)$.

Factors predicting overall survival were evaluated using univariate and multivariate analysis. In univariate analysis, PSADT $(p=0.021)$, PSAV $(p<0.001)$, Gleason score $(p=0.041)$, bone metastasis at diagnosis $(p<0.001)$, time to HRPCa development $(p<0.001)$, nadir PSA $(p=0.024)$ and PSA before diagnosis $(p<0.001)$ were statistically significant factors.

In multivariate analysis, PSAV $(p=0.007)$, bone metastasis at diagnosis $(p=0.001)$, and time to HRPCa development $(p=0.001)$ were found to be statistically significant in predicting overall survival, while other factors were found to be insignificant (Table III).

\section{DISCUSSION}

It has been reported that the serum PSA level observed after resistance to hormonal therapy increases with the development and the progression of metastatic disease and is related to the pathological stage of the disease. ${ }^{9}$ However, PSA is not sufficient to predict the response of the disease to treatment after appropriate treatment of patients with metastatic prostate cancer due to changes in its specific and laboratory conditions. Although PSA kinetics are used in the clinic at many stages from the diagnosis of prostate cancer to the planning of its treatment and even after the treatment, the studies on its clinical use in hormone-resistant prostate cancer are quite limited. Studies conducted to show whether PSA kinetics are independent prognostic factors in HRPCa are mostly studies that do not consider all kinetics at the same time. The clinical use of PSADT value was first described by D'Amico and Hanks in a study conducted in the early 1990s in which patients with local stage prostate cancer were examined. ${ }^{10}$ Partin et al. calculated the PSAV and tried to predict the presence of local recurrence or distant metastasis after radical prostatectomy. ${ }^{11}$

Studies conducted over the years among patients developing HRPCa and the current study show that PSA derived indicators can be an important predictive value for survival. ${ }^{12}$ In this study, the survival of those with lower PSADT values was found to be longer. Patients with PSADT>4.23 months had a mean survival of 2.2 times higher. This finding shows that PSADT can be an important predictive value for survival. The literature on this subject also supports this study. ${ }^{13}$ In a different study on the same subject, the PSADT during chemotherapy was calculated in patients with HRPCa development, and the relationship with the response to treatment was investigated. ${ }^{14}$ It was observed that the PSADT value was lower in those who did not respond to treatment.

In this study, because chemotherapy after HRPCa did not have a positive effect on survival, the authors could not examine the relationship between chemotherapy response and PSADT.

Another parameter that was statistically significant in predicting survival, like PSADT, is PSAV. In the current study, a low PSAV value was associated with a long follow-up time. It was observed that the mean survival of those with PSAV < $35.82 \mathrm{ng} / \mathrm{ml} /$ year was approximately two times higher. The subject of this study has not been adequately examined in the literature. A similar study excluded metastatic patients, while another included those treated with non-hormonal agents and found similar results to the present. ${ }^{15,16} \mathrm{~A}$ similar study on patients receiving chemotherapy also found results that support the present results. ${ }^{17}$

The time to reach HRPCa also appears as a different parameter in predicting survival in the present study. It was observed that the patients who developed HRPCa later, survived significantly longer. The role of the HRPCa development process in predicting survival is a parameter that has not been emphasised in studies so far. In this respect, this study points this an area that needs to be examined in the literature. Besides, this study examines many prognostic factors at the same time, and there are scarcely any publications in the literature on so many factors at the same time.

Studies are showing that there is a significant relationship between the time to HRPCa development and nadir PSA and the time to nadir PSA. ${ }^{18}$ These studies have shown that HRPCa development was later in those with low nadir PSA, and a long time to nadir PSA. In our study, the correlation between the time to nadir PSA and the time to develop HRPCa was evaluated and it was found that there was a significant difference in parallel with the literature.

On multivariate analysis, the relationship between the PSAV, bone metastasis, and time to HRPCa was found to be significant with overall survival. Additionally, it was observed that there was no significant difference in survival between patients who received chemotherapy after HRPCa and those who did not. These are findings that can be used as prognostic factors, and the results of our study are in line with the literature. ${ }^{19,20}$ The results of our study are valuable in terms of generating data for the clinician to comment on survival. 
The fact that factors such as the ECOG performance score, pain score, and the number of metastatic lesions in the bone, which were proven to predict prognosis in previous studies, could not be analysed in this study, is the main limitation of the study.

\section{CONCLUSON}

PSADT, PSAV, nadir PSA, time to nadir PSA, and time to HRPCa are entities that can be accepted as prognostic factors in patients with HRPCa. This study is one of the rare studies in which all of them are handled simultaneously. Using these entities, the clinician will have a chance to predict survival in men with HRPCa. Thus, if there is a treatment option to be arranged according to the survival, that will be given on a more reliable basis.

\section{ACKNOWLEDGEMENTS:}

We thank all patients who participated in the study and those who carried out the extensive clinical and laboratory work. We also thank Yavuz Selcuk Untan (English Language Teacher, Kayseri, Turkey) for translation.

\section{ETHICAL APPROVAL:}

All procedures performed in human participants were in accordance with the ethical standards of the Institutional and/or National Research Committee and with the 1964 Helsinki Declaration and its later amendments or comparable ethical standards. This study was conducted under the approval of the Ethical Council of Erciyes University, Faculty of Medicine (10.02.2012 / 2012-140).

\section{PATIENTS' CONSENT:}

Informed consents were obtained from all individual participants included in the study.

\section{CONFLICT OF INTEREST:}

The authors declared no conflict of interest.

\section{AUTHORS' CONTRIBUTION:}

VS: Conceived the study, collected data.

IU: Set up the study, analysed the data, wrote the manuscript.

AT: Provided supervision.

\section{REFERENCES}

1. Pakzad R, Mohammadian-Hafshejani A, Ghoncheh M, Pakzad I, Salehiniya $\mathrm{H}$. The incidence and mortality of prostate cancer and its relationship with development in Asia. Prostate Int 2015; 3(4):135-40. doi: 10.1016/j.prnil. 2015.09. 001.

2. Kurian CJ, Leader AE, Thong MSY, Keith SW, Zeigler-Johnson $\mathrm{CM}$. Examining relationships between age at diagnosis and health-related quality of life outcomes in prostate cancer survivors. BMC Public Health 2018; 18(1):1060. doi: 10. 1186/s12889-018-5976-6.

3. Trock BJ, Han M, Freedland SJ, Humphreys EB, DeWeese TL, Partin AW, et al. Prostate cancer-specific survival following salvage radiotherapy vs. observation in men with biochem- ical recurrence after radical prostatectomy. JAMA 2008; 299(23):2760-9. doi: 10.1001/jama.299.23.2760.

4. Hoimes CJ, Kelly WK. Redefining hormone resistance in prostate cancer. Ther Adv Med Oncol 2010; 2(2):107-23. doi: $10.1177 / 1758834009356433$.

5. Teo MY, Rathkopf DE, Kantoff P. Treatment of advanced prostate cancer. Annu Rev Med 2019; 70:479-99. doi: 10.1146/annurev-med-051517-011947.

6. Moul JW. The evolving definition of advanced prostate cancer. Rev Urol 2004; 6 Suppl 8(Suppl 8):S10-7.

7. Prcic A, Begic E, Hiros M. Usefulness of total PSA value in prostate diseases diagnosis. Acta Inform Med 2016; 24(3):156-61. doi: 10.5455/aim.2016.24.156-161.

8. Eisenhauer EA, Therasse P, Bogaerts J, Schwartz LH, Sargent $D$, Ford $R$, et al. New response evaluation criteria in solid tumours: Revised RECIST guideline (version 1.1). Eur J Cancer 2009; 45(2):228-47. doi: 10.1016/j.ejca.2008. 10.026.

9. Sasaki T, Sugimura $Y$. The importance of time to prostate-specific antigen (PSA) nadir after primary androgen deprivation therapy in hormone-naive prostate cancer patients. J Clin Med 2018; 7(12):565. doi: 10.3390/ jcm7120565.

10. Hanks GE, D'Amico A, Epstein BE, Schultheiss TE. Prostatic-specific antigen doubling times in patients with prostate cancer: A potentially useful reflection of tumor doubling time. Int J Radiat Oncol Biol Phys 1993; 27(1):125-7. doi: 10.1016/0360-3016(93)90429-y.

11. Partin AW, Pearson JD, Landis PK, Carter HB, Pound CR, Clemens JQ, et al. Evaluation of serum prostate-specific antigen velocity after radical prostatectomy to distinguish local recurrence from distant metastases. Urology 1994; 43(5):649-59. doi: 10.1016/0090-4295(94)90180-5.

12. O'Brien MF, Cronin AM, Fearn PA, Savage CJ, Smith B, Stasi J, et al. Evaluation of prediagnostic prostate-specific antigen dynamics as predictors of death from prostate cancer in patients treated conservatively. Int J Cancer 2011; 128(10):2373-81. doi: 10.1002/ijc.25570.

13. Semeniuk RC, Venner PM, North S. Prostate-specific antigen doubling time is associated with survival in men with hormone-refractory prostate cancer. Urology 2006; 68(3):565-9. doi: 10.1016/j.urology.2006.03.055.

14. Hanninen $M$, Venner $P$, North $S$. A rapid PSA half-life following docetaxel chemotherapy is associated with improved survival in hormone refractory prostate cancer. Can Urol Assoc J 2009; 3(5):369-74.

15. Thurtle D, Rossi SH, Berry B, Pharoah P, Gnanapragasam VJ. Models predicting survival to guide treatment decision-making in newly diagnosed primary non-metastatic prostate cancer: A systematic review. BMJ Open 2019; 9(6): e029149. doi: 10.1136/bmjopen-2019-029149.

16. Antonarakis ES, Zahurak ML, Lin J, Keizman D, Carducci MA, Eisenberger MA. Changes in PSA kinetics predict metastasis- free survival in men with PSA-recurrent prostate cancer treated with nonhormonal agents: Combined analysis of 4 phase II trials. Cancer 2012; 118(6):1533-42. doi: 10.1002/cncr.26437.

17. Daskivich TJ, Regan MM, Oh WK. Distinct prognostic role of 
prostate-specific antigen doubling time and velocity at emergence of androgen independence in patients treated with chemotherapy. Urology 2007; 70(3):527-31. doi: 10.1016/ j.urology.2007.04.035.

18. Tomioka A, Tanaka N, Yoshikawa M, Miyake M, Anai S, Chihara $Y$, et al. Nadir PSA level and time to nadir PSA are prognostic factors in patients with metastatic prostate cancer. BMC Urol 2014; 14:33. doi: 10.1186/1471-2490-
14-33.

19. Moreira DM, Howard LE, Sourbeer KN, Amarasekara HS, Chow LC, Cockrell DC, et al. Predicting time from metastasis to overall survival in castration-resistant prostate cancer: Results from search. Clin Genitourin Cancer 2017; 15(1):60-6 e2. doi: 10.1016/j.clgc.2016. 08.018.

20. Nader R, El Amm J, Aragon-Ching JB. Role of chemotherapy in prostate cancer. Asian J Androl 2018; 20(3):221-9. doi: 10.4103/aja.aja_40_17. 